\title{
The Joint Use of the Ordinary and Extraordinary Virtual Height Curves in Determining Ionospheric Layer Profiles
}

\author{
L. R. O. Storey ${ }^{1}$
}

(October 8, 1959)

\begin{abstract}
An extension of Budden's matrix method for determining ionospheric layer profiles is described. When analyzing vertical incidence ionograms by the matrix method, it is usual to interpret the virtual height curve for the ordinary mode only. Errors then arise from the presence in the lower ionosphere of low-density ionization for which the plasma frequency is less than the lowest frequency observed. In the proposed extension of the method, such errors are reduced by making use of the extraordinary virtual height curve as well as of the ordinary.
\end{abstract}

\section{Introduction}

In 1954 Budden [1] ${ }^{2}$ introduced the matrix method for analyzing the records of vertical incidence ionospheric soundings, and since then it has come into wide use. The records, which are known as "ionograms," contain curves of the virtual reflection heights of the ordinary and extraordinary modes as functions of frequency ("virtual height curves"), and the object of the analysis is to determine, from one or the other of these curves, how the ionization in the underside of the ionosphere is distributed in height. The difficulty lies in the integral equation that relates this distribution to the virtual height curve; in general, it is too complex to be solved analytically. The principle of Budden's method is to approximate the integral by a sum of discrete values of the integrand, thus converting the integral equation into a matrix equation, which may be solved by inverting the matrix.

In its customary form, the method makes use only of the ordinary virtual height curve. The ordinates of this curve are measured from the ionogram at selected frequencies, and then this set of ordinates is multiplied by a matrix to produce a set of the corresponding ordinates of the layer profile. The matrix multiplication is a simple repetitive process and so lends itself readily to performance by a digital computer. As a result, the matrix method is now widely favored for the routine analysis of ionospheric data.

Unfortunately, however, two rather restrictive conditions have to be met if the method is to work properly: First, the original virtual height curve should extend down to zero frequency; second, the ionosphere should consist of a single layer only, so that a given density of ionization occurs at just one height on its underside. Only under these conditions does the integral equation define a unique distribution.

1 Central Radio Propagation Laboratory, National Bureau of Standards, Boulder, Colo.; present address, Centre National de la Recherche Scientifique, Service d'A eronomie, Observatoire de Meudon (S,-\&-O.). Paris, France.

2 Figures in brackets indicate the literature references at the end of this paper.
Usually neither condition is met in practice. Most ionospheric sounders do not operate below about $1 \mathrm{Mc}$, while the ionosphere often appears to be stratified into several distinct lavers. Then there are many possible distributions that could have given rise to the observed portion of the virtual height curve. In fact, these uncertainties are the main sources of error in the analysis of ionograms by the matrix method.

This paper deals only with the problem of how to reduce the errors that arise from ignorance of the virtual heights at low frequencies. Here the ionosphere still is assumed to consist of a single layer. The analysis appears capable of being extended to cover the case of multiple layers with intervening "valleys," but this development is not considered.

When the virtual height curve is missing at the low-frequency end, the usual practice is to assume that the unknown virtual heights are constant and equal to the value at the lowest frequency observed; this assumption implies that there is no ionization below the level of reflection for that frequency. If such "low-density ionization" is present in fact, then the group delay that it produces in the observed frequency range is attributed wrongly to time spent by the waves in traveling up to the ionosphere, so that the true heights are overestimated.

So long as attention is confined to a single virtual height curve, there is no way of detecting the lowdensity ionization or of isolating its effects in the observed frequency range. But these effects are different for the two modes of propagation; the group delays differ in amount and vary with frequency in different ways. This fact suggests that the low-density ionization could be studied by measuring both the ordinary and extraordinary virtual height curves and combining the data that they contain. In the present paper, extensions of the Budden matrix method are developed to do this.

The contents of the paper are arranged as follows: In section 2 terms are defined, and the present method for the interpretation of a single virtual height curve 
is reviewed briefly; the work reviewed is chiefly that of Budden [1], Rydbeck [2], and Schmerling [3]. Section 3, which contains the substance of the paper, shows how the matrix method may be extended to make joint use of both virtual height curves; the errors that may arise in the inferred distribution of ionization, due to random errors in measuring the virtual heights, are examined also. The analysis is illustrated in section 4 by a worked example, in which an artificial ionogram, computed for a known distribution of ionization, is interpreted by the new procedures. Finally, the whole analysis is discussed and criticized in section 5, and the conclusions of the paper are summarized in section 6 .

Certain previous authors also have considered using the two virtual height curves in conjunction. The suggestion was made originally by Jackson [4], but his approach, which is one of trial and error, is not suitable for extensive use. Thomas [5], however, in a recent survey paper, has outlined some work by Titheridge [6], who appears to have adapted Jackson's method into a more convenient form. Titheridge's work, which has not been published at the time of writing this paper, may anticipate some of the results presented here.

\section{Interpretation of a Single Virtual Height Curve}

\subsection{The Integral Equations}

In this section the integral equations are derived that relate the virtual height curves for the ordinary and extraordinary modes to the distribution of ionization.

At vertical incidence, the virtual height of reflection $h^{\prime}$ is given as a function of the wave frequency $f$ by the integral

$$
h^{\prime}(f)=\int_{0}^{z_{R}} \mu^{\prime}(f, N) d z
$$

where $z$ is height in general, $z_{R}$ is the true height of reflection for the given frequency, and $\mu^{\prime}$ is the group refractive index, which depends both on the frequency and on the local electron density $N$.

In the limit of very low wave frequencies, the virtual and true heights of reflection for both modes all tend to a single limiting value $z_{0}$, the height of the base of the ionosphere. From ground level up to this height the waves travel at the speed of light, which is to say that their group refractive indices are unity. $^{3}$ Thus (1) can be written as

$$
h^{\prime}(f)=z_{0}+\int_{z_{0}}^{z_{R}} \mu^{\prime}(f, N) d z
$$

\footnotetext{
3 Actually $\mu^{\prime}$ is slightly greater than unity even below the ionosphere due to the refraction of the air, but the departure from unity is negligible in the present context.
}

where the integral now embraces only the range of height in which free electrons are present.

The general expression for the virtual height is completed by introducing the relation between the density of electrons and the height. However, instead of the density itself, it is more convenient to work with the "plasma frequency" $f_{N}$. which is proportional to its square root. The function $z\left(f_{N}\right)$, in which plasma frequency is viewed as the independent and height as the dependent variable, will be referred to here as the "layer profile." The profile is a multivalued function. even for a single layer, since a given value of $f_{N}$, if it occurs at all, must do so at least twice, once on the upper surface of the layer and once on its underside. The relationship between the true heights of reflection and the layer profile may be understood by considering the condition for reflection. The condition for an ionized medium to reflect radio waves is that its plasma frequency should exceed a certain critical value that depends on the frequency of the waves and also on the mode of propagation. This value will be written as $f_{R}(f)$. It follows that waves of a certain frequency and mode, propagated up into the ionosphere from below, are reflected at the first level that they encounter at which $f_{N}$ is equal to the appropriate value of $f_{R}$. Obviously, this is the lowest such level. Hence, the true height of reflection is given by

$$
z_{R}=\text { minimum value of } z\left(f_{R}\right)
$$

To summarize, the true height of reflection is a particular value of $z$, determined by $f_{R}$, which in turn is a function of $f$; this function is different for the two modes of propagation.

Since all reflection takes place from the underside of the ionosphere, only the profile of this part needs to be considered. Further, since the ionosphere has been assumed to consist of a single layer, the profile of its underside is a monotonic function. In this case, $f_{N}$ can be taken as the variable of integration. Also, the group refractive index $\mu^{\prime}$ will be expressed as a function of $f_{N}$ instead of $N$. With these changes, the integral equation becomes

$$
h^{\prime}(f)=z_{0}+\int_{0}^{f_{R}} \mu^{\prime}\left(f, f_{N}\right)\left(\frac{d z}{d f_{N}}\right) d f_{N} .
$$

Here the equation is still quite general, in that it applies equally to both modes. The particular equations for the ordinary and extraordinary modes are to be obtained by considering their respective reflection conditions, that is to say, the different relationships between $f_{R}$ and $f$. These relationships are graphed in figure 1.

The ordinary mode behaves very simply; the critical plasma frequency for reflection is equal to the wave frequency. Thus,

$$
f_{R}=f \quad \text { (ordinary mode) }
$$




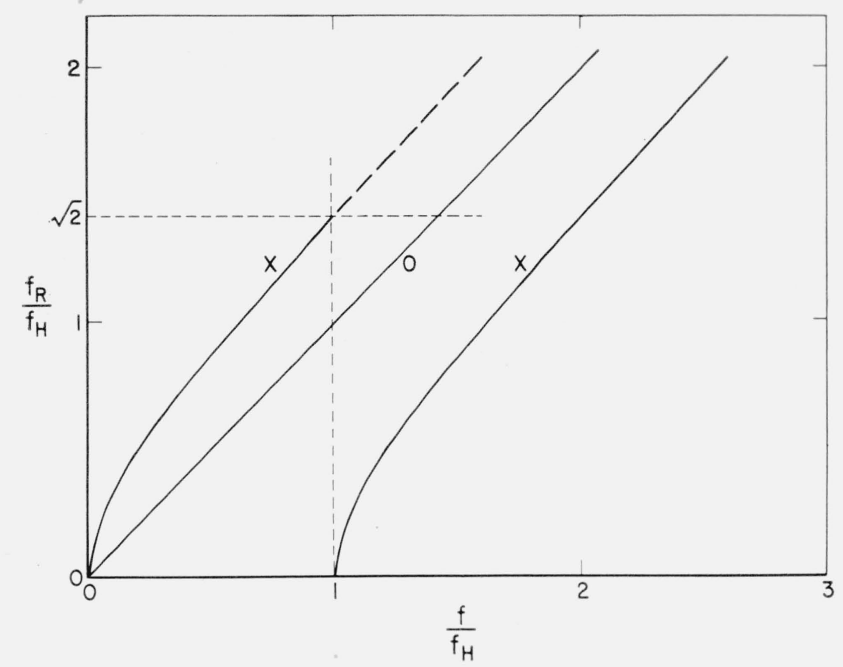

FIGURE 1. The conditions for reflection of the ordinary (O) and extraordinary (X) magneto-ionic modes at vertical incidence.

Abscissa: wave frequency $(f)$. Ordinate: plasma frequency at the level of reflection $\left(f_{R}\right)$. Both frequencies are normalized with respect to the gyrofrequency $\left(f_{H}\right)$.

so the integral equation for the ordinary mode is

$$
h^{\prime}(f)=z_{0}+\int_{0}^{f} \mu^{\prime}\left(f, f_{N}\right)\left(\frac{d z}{d f_{N}}\right) d f_{N} .
$$

From here on, the quantities $h, h^{\prime}$, and $\mu^{\prime}$, when written without subscripts, will refer to the ordinary mode only. The corresponding quantities for the extraordinary mode will bear the subscript " $x . "$

The extraordinary reflection condition is more complex. The relationship between $f_{R}$ and $f$ is now

$$
\begin{aligned}
f_{R} & =\left\{f\left(f \pm f_{H}\right)\right\}^{\frac{1}{2}} \quad \text { (extraordinary mode) } \\
& =\tilde{f}, \quad \text { say }
\end{aligned}
$$

where $f_{H}$ is the gyrofrequency. The positive sign applies if $f<f_{H}$ (L.H. curve in fig. 1), while the negative sign applies in the more usual situation, where $f>f_{H}$ (R.H. curve).

Note that, as the wave frequency approaches the gyrofrequency from below, $f_{R}$ tends to the value $\sqrt{2}$ $t_{H}$. On the other hand, as the gyrofrequency is approached from above, $f_{R}$ becomes vanishingly small, so that the extraordinary reflection level should descend to the base of the ionosphere; actually, such echoes are never detected, because they are heavily absorbed.

The integral equation for the extraordinary mode can be written now as

$$
h_{x}^{\prime}(f)=z_{0}+\int_{0}^{\tilde{f}} \mu_{x}^{\prime}\left(f, f_{N}\right)\left(\frac{d z}{d f_{N}}\right) d f_{N},
$$

where the symbol $\tilde{f}$ has been adopted to denote the value of $f_{R}$ for an extraordinary wave of frequency $f$.
This equation differs slightly from the corresponding equation for the ordinary mode, in that the upper limit of the integral $(\tilde{f})$ is not the same as the independent variable $(f)$ on the left-hand side; also, to each value of $f$ below the limit $\sqrt{2} f_{H}$ there correspond two possible values of $f$ (see fig. 1 ), one of which is less than the gyrofrequency, while the other is greater. However, only the upper range of $f$ will be considered here, because most records of ionospheric virtual height do not extend below the gyrofrequency. With this restriction, there is a straightforward correspondence between $\tilde{f}$ and $f$, and the virtual height may be regarded equally well as a function of either variable. When it is regarded as a function of $\tilde{f}$, the extraordinary virtual height will be represented by the symbol $\tilde{h}$; thus

$$
\tilde{h}(\tilde{f})=h_{x}^{\prime}(f) .
$$

To find the virtual height for a specified value of $\tilde{f}$, it is necessary to know the corresponding value of $f$. This frequency is given by the positive solution of the quadratic eq (7), and it is

$$
f=\frac{1}{2} f_{H}\left\{\left\{1+4\left(\tilde{f} / f_{H}\right)^{2}\right\}^{\frac{1}{2}} \mp 1\right\}
$$

where the positive sign now yields the range of frequencies above $f_{H}$, which is the range to be considered.

In these terms, the integral equation for the extraordinary mode is

$$
\tilde{h}(\tilde{f})=z_{0}+\int_{0}^{\tilde{f}} \mu_{x}^{\prime}\left(f, f_{N}\right)\left(\frac{d z}{d f_{N}}\right) d f_{N} .
$$

This equation is similar to (6), the only remaining difference being that, in calculating $\mu_{x}^{\prime}$, it is necessary first to obtain from (11) the value of $f$ that corresponds to the given value of $\tilde{f}$.

The integral eqs (6) and (12) relate the virtual height curves to the slope of the layer profile. Were it possible to invert either equation so as to obtain a solution for the slope, then the profile itself could be recovered by a straightforward integration.

\subsection{The Matrix Equations}

The integral equations cannot be solved analytically in the general case, because the group refractive indices are complicated functions of $f$ and $f_{N}$. Therefore, they are solved by converting them into matrix equations as will be described now.

Consider the eq (6) for the ordinary mode. The first step is to replace the continuous functions $h^{\prime}$ and $z$ by their values at a number of discrete "sample frequencies." These frequencies will be written, in order of ascending magnitude, as $f_{0}, f_{1}, f_{2} \ldots$. etc., or generally as $f_{m}$, where the index $m$ is zero or a 
positive integer; $f_{0}$ is zero frequency, and the rest are assumed to be spaced closely, but not necessarily evenly, through the full frequency range. The following notation will be used also:

$$
\begin{aligned}
\Delta f_{m} & =f_{m}-f_{m-1} \quad(m \geqq 1) \\
h_{n}^{\prime} & =h^{\prime}\left(f_{n}\right) \\
z_{m} & =z\left(f_{m}\right) \\
\Delta f_{m} & = \begin{cases}z_{m}-z_{m-1} & (m \geqq 1) \\
z_{0} & (m=0) .\end{cases}
\end{aligned}
$$

The quantities $\Delta z_{m}$ are increments of height on the layer profile; note the definition for $m=0$.

The next step in the approximation is to assume that the slope of the layer profile is effectively constant over each elementary frequency interval $\Delta f_{m}$, i.e.,

$$
\frac{d z}{d f_{N}} \simeq \frac{\Delta z_{m}}{\Delta f_{m}} \quad\left(f_{m-1} \leqq f_{N} \leqq f_{m}\right)
$$

Then the complete integral can be split up into a series of discrete sums, as follows:

$$
\begin{aligned}
h_{n}^{\prime} & \simeq \Delta z_{0}+\sum_{m=1}^{n} \frac{\Delta z_{m}}{\Delta f_{m}} \int_{f_{m-1}}^{f_{m}} \mu^{\prime}\left(f_{n}, f_{N}\right) d f_{N} \\
& \simeq \sum_{m=0}^{n} M_{n m} \Delta z_{m}, \quad \text { say }
\end{aligned}
$$

where $\Delta z_{0}$ has been written in place of $z_{0}$, and

$$
M_{n m}= \begin{cases}1 & (m=0) \\ \frac{1}{\Delta f_{m}} \int_{f_{m-1}}^{f_{m}} \mu^{\prime}\left(f_{n}, f_{N}\right) d f_{N} & (1 \leqq m \leqq n) \\ 0 & m>n)\end{cases}
$$

Now suppose that in (17), $n$ is set equal in turn to $0,1,2$, etc., up to the value that corresponds to the highest sample frequency. The result is an array of simultaneous equations, which may be solved for the unknowns $\Delta z_{m}$ by the simple algebraic process that is described below in section 2.4. Originally, however, Budden [1] adopted an alternative approach, which is to regard the complete array as a single "matrix equation;" this approach has provided a more convenient basis for the subsequent extensions of Budden's methods. In matrix terminology, the set of quantities $h_{n}^{\prime}$ and also the set of $\Delta z_{m}$, both constitute "vectors," while the coefficients $M_{n m}$ form a "lower triangular matrix." This differs from the corresponding matrix used by Budden (1955) and Schmerling (1957) by including an extra column with $m=0$, in which all the coefficients are unity, and an extra row with $n=0$, in which all the coefficients except the first are zero. Each of the remaining nonzero coefficients is the average of the values of group refractive index assumed by a wave of frequency $f_{n}$ in traveling from the level where $f=f_{m-1}$ up to that where $f_{N}=f_{m}$.

If $n$ is understood to run through its full range of values, then eq (17) states that the vector of the $h_{n}^{\prime}$ is obtained by multiplying the vector of the $\Delta z_{m}$ by the matrix of the coefficients $M_{n m}$. Thus (17) is the required matrix equation for the ordinary mode.

The integral equation for the extraordinary mode can be treated likewise, and the result is the matrix equation

$$
\tilde{h}_{n} \simeq \sum_{n=0}^{n} \tilde{M}_{n m} \Delta z_{m}
$$

where

$$
\tilde{M}_{n m}= \begin{cases}1 & (m=0) \\ \frac{1}{\Delta \tilde{f}_{m}} \int_{\tilde{f}_{m-1}}^{\tilde{f}_{m}} \mu_{x}^{\prime}\left(f_{n}, f_{N}\right) d f_{N} & (1 \leqq m \leqq n) \\ 0 & (m>n)\end{cases}
$$

and the other new quantities are defined like their ordinary counterparts (cf. eqs (13), (14)).

From here on, the complete matrices will be represented by symbols in square brackets, while the same symbols without brackets will refer to the individual elements. Thus the group refractive index matrices will be written as $\left[M_{n m}\right]$ and $\left[\tilde{M}_{n m}\right]$. Their elements $M_{n m}$ and $\tilde{M}_{n m}$ may be computed from the formulas given in appendix $\mathrm{A}$.

\subsection{Solution by Matrix Inversion}

The matrix eqs (17) and (19) for the ordinary and extraordinary modes, respectively, may be solved by inverting the matrices. Thus, for the ordinary mode, the solution is

$$
\Delta z_{n} \simeq \sum_{m=0}^{n} N_{n m} h_{m}^{\prime}
$$

where the matrix $\left[N_{n m}\right]$ is the inverse of $\left[M_{n m}\right]$. Similarly, for the extraordinary mode,

$$
\Delta z_{n} \simeq \sum_{m=0}^{n} \tilde{N}_{n m} \tilde{h}_{m}
$$

where $\left[\tilde{N}_{n m}\right]$ is the inverse of $\left[\tilde{M}_{n m}\right]$. Because the matrices $\left[M_{n m}\right]$ and $\left[\tilde{M}_{n m}\right]$ are lower triangular, so also are their inverses.

The increments of height may be summed now to give the heights themselves:

$$
z_{n}=\sum_{m=0}^{n} \Delta z_{m} .
$$

This summation is analogous to the final integration that would be performed if the original integral equations, that involve the slope $d z / d f_{N}$, could be solved analytically (see sec. 2.1). 


\subsection{Solution Row-by-Row}

As an alternative to inverting the matrices, the increments of height on the layer profile may be found by solving the matrix equation row-by-row [7]. For instance, consider the eq (17) for the ordinary mode. It can be rearranged to read

$$
\Delta z_{n} \simeq \frac{1}{M_{n n}}\left\{h_{n}^{\prime}-\sum_{m=0}^{n-1} M_{n m} \Delta z_{m}\right\}
$$

There is one such equation for each value of $n$. The complete set may be solved by starting from $n=0$ and working upwards. Thus,

$$
\begin{aligned}
& \Delta z_{0} \simeq \frac{1}{M_{00}}\left\{h_{0}^{\prime}\right\} \\
& \Delta z_{1} \simeq \frac{1}{M_{11}}\left\{h_{1}^{\prime}-M_{10} \Delta z_{0}\right\}, \\
& \Delta z_{2} \simeq \frac{1}{M_{22}}\left\{h_{2}^{\prime}-M_{21} \Delta z_{1}-M_{20} \Delta z_{0}\right\}
\end{aligned}
$$

and so on. In effect, the method consists of isolating, at each stage, the group delay produced in the unknown uppermost increment of height, by subtracting from the observed virtual height the group delay produced in the known height increments at lower levels.

The eq (19) for the extraordinary mode can be solved similarly. Matrix equations can be solved in this way only when, as in the present case, the matrices are triangular.

\section{Joint Use of Both Virtual Height Curves}

\subsection{General Remarks}

In the previous section, each of the matrix eqs (17) and (19) represented a set of linear simultaneous equations in which the number of data (ordinates of the virtual height curve) was equal to the number of unknowns (increments of height on the layer profile). Hence each set had an exact solution, so that the complete virtual height curve for either mode was sufficient by itself to determine the profile. But when some of the curve is missing, as when the records are unavailable at low frequencies, then the remaining high frequency data are insufficient to determine the profile without making special assum.ptions (see sec. 1).

However, the situation is not hopeless, since the data for the two magneto-ionic modes are mutually independent. Suppose, therefore, that in sampling the virtual height curves to obtain these data, the sample frequencies are chosen so that the unknowns are the same in the two sets of equations. Then, if both sets are combined, the number of data is increased while the number of unknowns remains constant. Hence, the total data may now be enough for the complete set of equations to be soluble.

To make the unknowns the same, the ordinary and extraordinary virtual height curves must be sampled at frequencies which correspond to the same true heights of reflection. Considering one such pair of frequencies, this is to say that the values of $f_{R}$ must be the same for the two modes. From the expressions for $f_{R}((5)$ and $(7))$, this requirement is seen to be met if the sample frequency $f_{n}$ for the ordinary mode is made equal to $\tilde{f}_{n}$ for the extraordinary; the corresponding sample frequency $f_{n}$ for the extraordinary may be found from (11). Thus the corresponding values of $f_{n}$ are not equal for the two modes; moreover, if the sample frequencies are spaced evenly for the ordinary mode, then those for the extraordinary mode will be spaced unevenly. From here on, the two sets of sample frequencies will be assumed to be related in this way.

The question now arises, under what condition can the layer profile be determined fully by joint use of both virtual height curves? Suppose, to simplify the discussion, that all the data come in pairs; sample values of ordinary virtual height for which no corresponding extraordinary virtual heights are measurable, or vice versa, will be ignored. Suppose also that the range of index $n$ for the two sets of data is $r \leqq n \leqq r+s-1$. Then the data comprise $s$ values of $h_{n}^{\prime}$, together with $s$ values of $\tilde{h}_{n}$, a total of $2 s$ separate items. The number of the unknowns $\Delta z_{m}$ is $r+s$. Evidently the condition ${ }^{4}$ under which the data determine the unknowns fully is that $s \geqq r$.

If the positions of the pairs of sample frequencies can be chosen arbitrarily, it is easy to arrange for a surplus of data. If, on the other hand, it is decided to space the frequencies evenly for one or other of the modes, say the ordinary, then the condition entails that the frequency range over which the ordinary virtual height is measurable should be wider than the unobserved range of low frequencies. Since, for most ionosondes, the low frequency limit lies below $2 \mathrm{Mc}$, while the ordinary penetration frequency for the $F 2$ layer usually exceeds $4 \mathrm{Mc}$, this requirement is likely to be met most of the time.

Assume, then, that there are enough data to determine the unknowns. Two distinct cases are to be considered: In the first, $s$ is equal to $r$, so that the data determine the unknowns exactly; in the second, $s$ is greater than $r$, and the unknowns are over-determined. The exactly-determined case is treated in section 3.2 , and the overdetermined case in section 3.3 .

\footnotetext{
${ }^{4}$ Strictly speaking, this condition is necessary but not sufficient, for, even when it is satisfied, the determinant of the set of equations still might vanish. In practice, the determinant is unlikely to vanish exactly, though it might become very small. Then quite small errors in the data would produce large errors in the inferred values of the unknowns, a state of affairs that is to be avoided. For
} a discussion of the effects of random errors, see section 3.5. 


\subsection{The Exactly-Determined Case}

In the exactly-determined case, the index $n$ for the data $\left(h_{n}^{\prime}\right.$ and $\left.\tilde{h}_{n}\right)$ runs from $r$ to $2 r-1$. The matrix eqs (17) and (19) together represent a set of $2 r$ simultaneous equations, which are to be solved for the $2 r$ unknown values of the $\Delta z_{m}$.

It is convenient to split the solution into two steps: First, the equations are solved for the increments of height in the unobserved frequency range $(0 \leqq m<r)$; after which, the remaining increments in the observed range $(r \leqq m \leq 2 r-1)$ are found by existing methods.

In finding the $\Delta z_{m}$ for the range $0 \leqq m<r$, the values outside this range first must be eliminated. This is done most simply by starting from the inverse eqs (21) and (22). Because the sample frequencies for the ordinary and extraordinary virtual height curves correspond to the same true heights of reflection, these two expressions must give the same values for the $\Delta z_{n}$ to the limits of accuracy of the matrix formulas. Therefore, they can be equated to yield the relation that holds between the two virtual height curves as a consequence of their being derived from the same layer profile:

$$
\sum_{m=0}^{n}\left\{\tilde{N}_{n m} \tilde{h}_{n}-N_{n m} h_{m}^{\prime}\right\} \simeq 0
$$

When $n \geqq r$, (25) provides a relation between the observed and the unobserved virtual heights. This fact can be made plain by splitting the sum into separate parts for the two frequency ranges:

$$
\sum_{m=r}^{n}\left\{N_{n m} h_{m}^{\prime}-\tilde{N}_{n m} \tilde{h}_{m}\right\} \simeq \sum_{m=0}^{r-1}\left\{\tilde{N}_{n m} \tilde{h}_{m}-N_{n m} h_{m}^{\prime}\right\}(n \geq r) .
$$

The next step is to substitute for the unobserved virtual heights and reintroduce the corresponding $\Delta z_{m}$ by use of (17) and (19):

$$
\begin{aligned}
\sum_{m=r}^{n}\left\{N_{n m} h_{m}^{\prime}-\tilde{N}_{n m} \tilde{h}_{m}\right\} & \simeq \sum_{m=0}^{r-1}\left\{\tilde{N}_{n m}\left(\sum_{l=0}^{m} \tilde{M}_{m l} \Delta z_{l}\right)\right. \\
& \left.-N_{n m}\left(\sum_{l=0}^{m} M_{m l} \Delta z_{l}\right)\right\} \quad(n \geq r)
\end{aligned}
$$$$
\simeq \sum_{m=0}^{r-1} \sum_{l=0}^{m}\left\{\tilde{N}_{n m} \tilde{M}_{m l}-N_{n m} M_{m l}\right\} \Delta z_{l} \quad(n \geq r)
$$$$
\simeq \sum_{l=0}^{r-1} \sum_{m=l}^{r-1}\left\{\tilde{N}_{n m} \tilde{M}_{m l}-N_{n m} M_{m l}\right\} \Delta z_{l} \quad(n \geq r)
$$

The reversal of the order of summation on the right-hand side, between (27) and (28), may be justified by pointing out that, in the form of (27) a given $m$ occurs in combination with all the l's from 0 to $m$; hence, a given $l$ must occur in combination with all the $m$ 's from $l$ to $r-1$, as indicated in
(28). Or again, it may be checked simply by writing down the triangular array of the possible combinations of $l$ and $m$.

Now (28) may be written in the concise form

$$
S_{n} \simeq \sum_{l=0}^{r-1} P_{n l} \Delta z_{l} \quad(n \geq r)
$$

where

$$
S_{n}=\sum_{m=r}^{n}\left\{N_{n m} h_{m}^{\prime}-\tilde{N}_{n m} \tilde{h}_{m}\right\} \quad(n \geq r)
$$

and

$$
P_{n l}=\sum_{m=l}^{r-1}\left\{\tilde{N}_{n m} \tilde{M}_{m l}-N_{n m} M_{m l}\right\} \quad(n \geq r)
$$

The matrix eq (29) relates the $\Delta z_{l}$ for the unobserved frequency range to the quantities $S_{n}$, which are formed from pairs of corresponding values of ordinary and extraordinary virtual height in the observed range. Its solution will be written as

$$
\Delta z_{l} \simeq \sum_{n=r}^{2 r-1} Q_{l n} S_{n} \quad(l \leq r-1)
$$

where the matrices $\left[Q_{l n}\right]$ and $\left[P_{n l}\right]$ are mutually inverse.

Having thus arrived at the height increments $\Delta z_{\text {l }}$ for $l \leq r-1$, the remaining values can be found by solving one of the original matrix equations row-byrow (see sec. 2.4). Use of the virtual height data for either mode should lead to identical results, even when the data contain errors of measurement, because the equations for the two modes together form an exactly-determined set.

\subsection{The Overdetermined Case}

In the overdetermined case, where $s>r$, any subset of $r+s$ selections from the $2 s$ items of data would suffice, in principle, to determine the unknowns completely. If the data were free from error, it would not matter which subset was chosen. But when the data contain errors of measurement, some subsets will give better estimates than others. A still better estimate might be obtained by taking an appropriately weighted mean of the estimates from all the possible subsets, in which case each unknown would be given as a linear combination of all the data. The choice of the best combination would be governed by the statistics of the errors. Here it will be assumed, for convenience, that the errors of measurement of the virtual heights are all random, mutually uncorrelated, and distributed normally with equal standard deviations. Then the best estimate of the $\Delta z_{l}$ is that given by the "Method of Least Squares" (see, for instance, Whittaker and Robinson [8]), and it is the solution of the matrix equation

$$
R_{m} \simeq \sum_{l=0}^{r+s-1} U_{m l} \Delta z_{l}
$$


where

$$
R_{m}=\sum_{n=r}^{r+s-1}\left\{M_{n m} h_{n}^{\prime}+\tilde{M}_{n m} \tilde{h}_{n}\right\}
$$

and $\left[U_{m l}\right]$ is a square symmetric matrix, the elements of which are given by the expression

$$
U_{m l}=\sum_{n=r}^{r+s-1}\left\{M_{n m} M_{n l}+\tilde{M}_{n m} \tilde{M}_{n l}\right\}
$$

These equations are derived in appendix B.

The matrix eq (33) represents a new set of $r+s$ linear simultaneous equations, equal in number to the unknowns; each of the new equations is a linear combination of the $2 s$ original equations, represented by (17) and (19). The solution of the complete set will be written, in matrix form, as

$$
\Delta z_{l} \simeq \sum_{m=0}^{r+s-1} V_{l m} R_{m}
$$

where the matrix $\left[V_{l m}\right]$ is the inverse of $\left[U_{l m}\right]$ and likewise is square symmetric.

In eqs (33) to (36), the index $r$ would be a constant, fixed by the properties of the recording equipment, but the index $s$ would vary from one set of data to another because of the variations in the penetration frequencies of the F2 layer. Equation (36) is the solution of (33) just so long as $s \geq r$. The equations do not contain this restriction explicitly, but when $s<r$ the determinant of $\left[U_{m l}\right]$ vanishes so that the matrix cannot be inverted. Equation (36) is indeed applicable to the exactly-determined case when $s=r$, but for this special case the equations developed in section 3.2 give the same answer more simply; at least, the matrix that has to be inverted is smaller.

\subsection{Some Practical Considerations}

The variability of the index $s$ makes the full leastsquares solution inconvenient for practical use because each value of $s$ requires a different matrix $V_{l m}$ to be computed. In practice, it is best to fix $s$ at some particular value, so that $V_{l m}$ need be computed only once. The chosen value of $s$ should be sufficiently high for the unknowns to be thoroughly overdetermined, yet sufficiently low for the uppermost sample frequency $\left(f_{r+s-1}\right)$ never to exceed $f_{o} F_{2}$. The use of this particular matrix $\left[V_{l m}\right]$ yields the values of the $\Delta z_{l}$ for $0 \leqq l \leqq r+s-1$. When data are available at frequencies above $f_{r+s-1}$, the remaining $\Delta z_{l}$ may be obtained by continuing the row-byrow solution upwards. If the data contain random errors, however, the results would be different according as the ordinary or extraordinary virtual heights were used in the solution. Better results would be obtained by combining both sets of virtual height data following the Method of Least Squares. The least-squares value for each of these $\Delta z_{l}(l \geq r+s)$ is found by applying the procedure of section 2.4 to the equation

$$
\begin{aligned}
\Delta z_{l} \simeq \frac{M_{l l}}{M_{l l}^{2}+\tilde{M}_{l l}^{2}} & \left\{h_{l}^{\prime}-\sum_{m=0}^{l-1} M_{l m} \Delta z_{m}\right\} \\
& +\frac{\tilde{M}_{l l}}{M_{l l}^{2}+\tilde{M}_{l l}^{2}}\left\{\tilde{h}_{l}-\sum_{m=0}^{l-1} \tilde{M}_{l m} \Delta z_{m}\right\}
\end{aligned}
$$

which replaces (24).

Probably not much accuracy is lost by fixing the value of $s$ in the way suggested, which entails that only data at frequencies between $f_{r}$ and $f_{r+s-1}$ are used to determine the distribution of the lowdensity ionization. For this ionization has its greatest effects on the observed virtual heights at frequencies just above $f_{\tau}$, and hence these frequencies must provide the data that are most useful in determining its distribution.

Furthermore, the virtual height data at the higher frequencies may be systematically unreliable as a result of "lateral deviation." This effect is discussed more fully in section 5. Here it is mentioned only as a further justification for omitting the data at frequencies above $f_{\tau+s-1}$ initially, when using the matrix method for the overdetermined case.

\subsection{The Effect of Random Errors}

If the measurements of the virtual heights contain random errors, these errors produce fluctuations in the inferred values of the $\Delta z_{l}$. The standard deviation of these fluctuations will be calculated now for the total range of $l$. The calculation will be made for the overdetermined case assuming that the $\Delta z_{l}$ have been obtained by the full least-squares procedure ((33) to (36)). As before, the errors in the measurements will all be assumed to have the same standard deviation, and this will be called $\sigma$.

Now the change that occurs in $\Delta z_{l}$, as a result of a small error $\delta h_{n}^{\prime}$ in the measurement of the ordinary virtual height $h_{n}^{\prime}$, is found by differentiating (36) and (34):

$$
\begin{aligned}
\delta\left(\Delta z_{l}\right) & \simeq \sum_{m=0}^{r+s-1} V_{l m}\left(\frac{\partial R_{m}}{\partial h_{n}^{\prime}}\right) \delta h_{n}^{\prime} \\
& \simeq \sum_{m=0}^{r+s-1} V_{l m} M_{n m} \delta h_{n}^{\prime} .
\end{aligned}
$$

An error in the measurement of the extraordinary virtual height $\tilde{h}_{n}$ has a similar effect. The total standard deviation of $\Delta z_{l}$, due to all the errors, is found by squaring and adding these two contributions, summing over all $n$, averaging, and then taking the square root. The results can be written as:

$$
\text { Standard deviation of } \Delta z_{l}=\sigma \rho_{l} \text {, }
$$


where

$$
\rho_{l}^{2}=\sum_{n=r}^{r+s-1}\left\{\left[\sum_{m=0}^{r+s-1} V_{l m} M_{n m}\right]^{2}+\left[\sum_{m=0}^{r+s-1} V_{l m} \tilde{M}_{n m}\right]^{2}\right\} .
$$

The "error coefficient" $\rho_{l}$, which is the standard deviation of $\Delta z_{l}$ per unit standard deviation of the original measurements, is a measure of the susceptibility of the estimate of $\Delta z_{l}$ to disturbance by random errors of measurement; the smaller is $\rho_{l}$, the more reliable is the estimate.

For the exactly-determined case, the error coefficients may be obtained by using (40) with $s$ set equal to $r$. The special equations developed for this case in section 3.2 do not lend themselves well to the derivation of the coefficients.

Let it be emphasized that the $\rho_{l}$ are the error coefficients of the increments of height $\left(\Delta z_{l}\right)$ on the layer profile. Of greater interest, perhaps, are the error coefficients of the heights $\left(z_{l}\right)$ themselves. These coefficients will be called $\pi_{l}$, and they are defined thus, by analogy with the $\rho_{l}$ :

$$
\text { Standard deviation of } z_{l}=\sigma \pi_{l} \text {. }
$$

The $\pi_{l}$ cannot be derived from the $\rho_{l}$ in any simple way because the errors in the calculated $\Delta z_{l}$ are mutually correlated even though there is supposed to be no correlation between the errors in the virtual height data. An expression may be derived for the $\pi_{l}$, however, similarly to that for the $\rho_{l}$, using (23) as well as (36) and (34); it is

$$
\begin{aligned}
\pi_{l}^{2}=\sum_{n=r}^{r+s-1}\left\{\left[\sum_{k=0}^{l} \sum_{m=0}^{r+s-1} V_{k m} M_{n m}\right]^{2}\right. \\
\left.+\left[\sum_{k=0}^{l} \sum_{m=0}^{r+s-1} V_{k m} \tilde{M}_{r m}\right]^{2}\right\} .
\end{aligned}
$$

Alternatively, in appendix $\mathrm{C}$ it is shown that the whole analysis can be developed from the start in terms of the $z_{l}$ rather than the $\Delta z_{l}$, without altering the form of most of the expressions. In the analysis so developed, the $\pi_{l}$ would be given by the equation analogous to (40).

\section{A Test of the Method}

The foregoing analysis has been tested on an artificial ionogram. The test consisted of assuming a particular layer profile, computing the corresponding ionogram (fig. 2), and then attempting to recover the profile from the data in the ionogram, using first the conventional matrix method, and then the various extensions of the method that were developed in section 3. In the numerical calculations, the gyrofrequency was taken as $1450 \mathrm{kc}$, and the angle of dip as $68.2^{\circ}$, which are the values that apply at

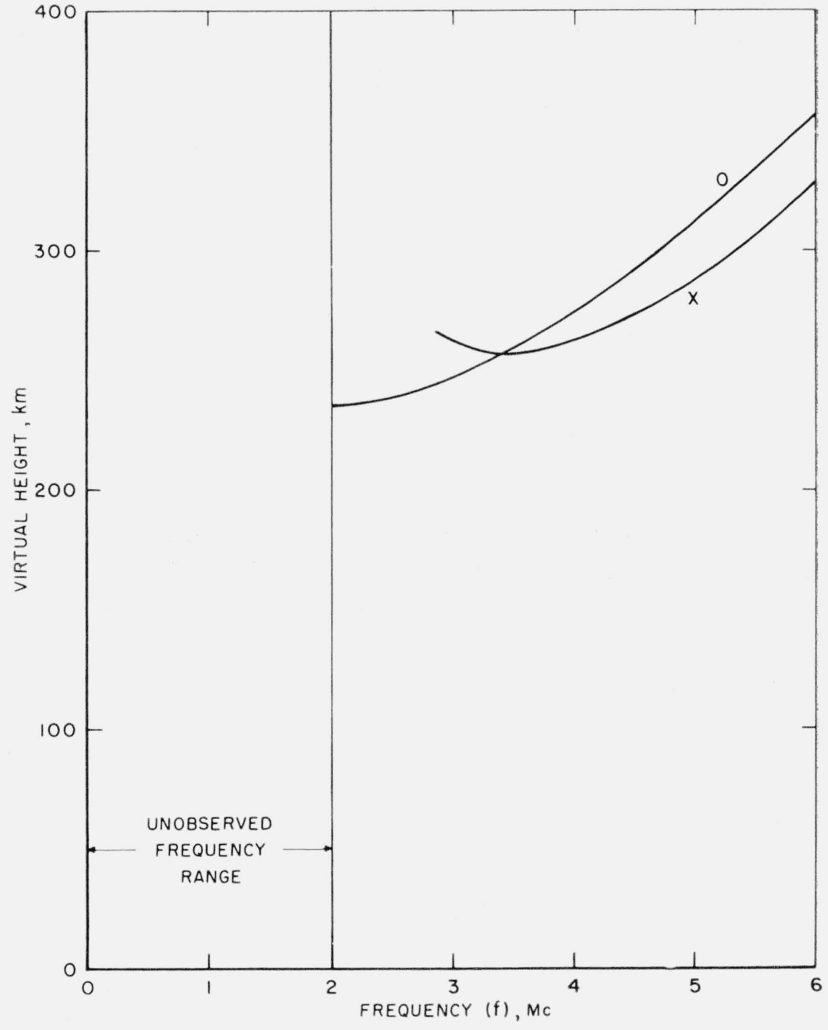

Figure 2. The virtual height curves calculated for the assumed layer profile.

Boulder, Colo. The calculations were performed on the IBM-650 computer at the Central Radio Propagation Laboratory of NBS.

The assumed layer profile is graphed as the solid curve in figures 3 to 5 . It is monotonic, since the analysis is restricted to profiles of this type for the time being. It represents a distribution of ionization in which there is a ledge of low density beneath a layer of high density. Such a distribution might arise in the ionosphere soon after dusk, when the E-layer is decaying rapidly but the $F$-layer persists.

The virtual height curves that are derived from this profile are shown in the "ionogram" of figure 2. The lowest observable frequency $\left(f_{r}\right)$ for the ordinary virtual height curve has been taken as $2 \mathrm{Mc}$, while the corresponding lower limit for the extraordinary curve is $2.85 \mathrm{Mc}$. The figure shows only those portions of the curves that are supposed to be observable. A particularly precise type of numerical integration (a 48-point Legendre-Gauss quadrature [9]) was used in computing the virtual heights, so that these data essentially are free from error. Hence any errors that may arise in the deduced layer profiles represent systematic defects in the methods of analysis.

The results of various attempts to recover the profile are presented in figures 3 to 5 . In these figures, the points on the deduced profile are shown as solid dots, while the broken line is a direct interpolation between adjacent points. 
Figure 3 shows the results of analyzing the ionogram by the conventional matrix method in which only the ordinary virtual height data are used [1, 3]. Because the low-density ionization has been ignored, all the computed points are much in error, lying well above the true profile (the solid curve); the error amounts to more than $30 \mathrm{~km}$ at the lowest observed frequency $(2 \mathrm{Mc})$, while even at $6 \mathrm{Mc}$ it is about $6 \mathrm{~km}$. It is this error that the extensions of the matrix method seek to eliminate.

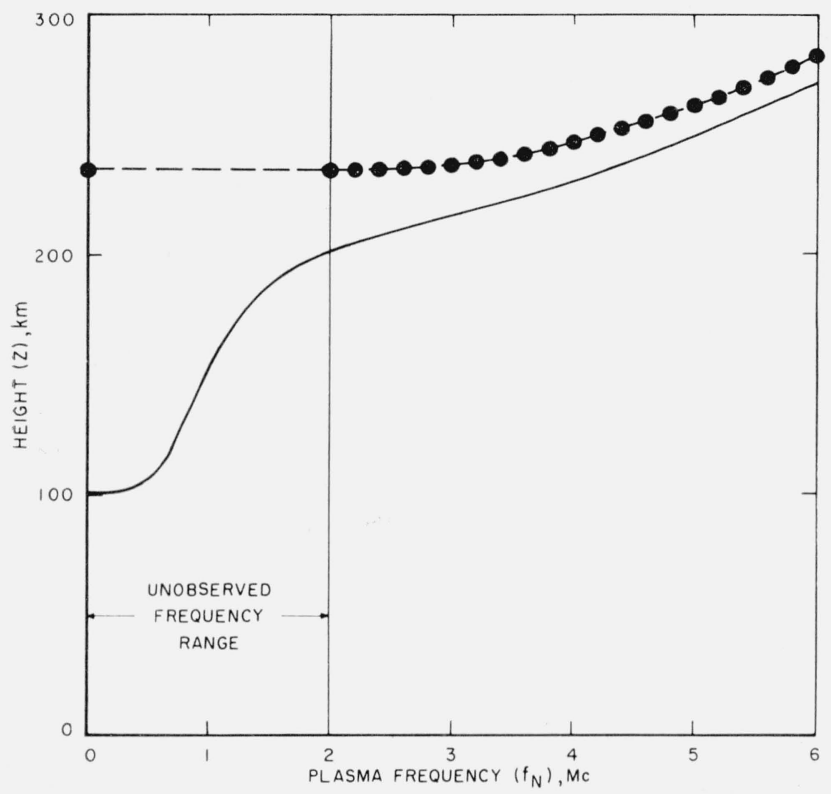

Figure 3. The layer profile inferred from analysis of the ordinary virtual height curve by the conventional matrix method.

For further explanation, see section 4 of the text.

However, in attempting to apply the new methods various problems were encountered, as follows:

The first, a comparatively trivial problem, was presented by the "rounding-off errors" in the digital computer. Such errors proved to be particularly serious in the inversion of the matrix $\left[U_{l m}\right]$. Eventually they were made negligible by performing the inversion with a "double-precision" program in which the computer carried 18 significant digits of each number instead of the usual 8 [10].

The main problem proved to be one of balance between the effects of random and systematic errors on the derived layer profile. The random errors are introduced by the inaccuracy of the virtual height data, as explained previously. The systematic errors are introduced when, in the process of converting the original integral equations into the corresponding matrix equations, the continuous curve of the profile is approximated by a series of rectilinear segments (see sec. 2.2.). The need to compromise between the two types of error does not arise in the analysis of a single virtual height curve, that is, in the situation studied in section 2 , for in that situation the systematic errors can be made arbitrarily small by increasing the number of sample frequencies and reducing the intervals between them. The introduction of a new sample frequency adds a new unknown to the set of equations, but it also adds a new item of data that is related closely to this particular unknown, so that the random errors are relatively unaffected. The situation is quite different, however, when the virtual height curves are incomplete. Then the introduction of a new sample frequency in the unobserved range increases the number of unknowns without adding to the data, so that the precision with which the profile can be determined, in the presence of random errors, suffers accordingly. It is clear that the disposition of the sample frequencies, particularly of those in the unobserved frequency range, is a delicate problem and one that involves balancing the effects of random and systematic errors against each other. In view of the complexity of the equations, it seemed futile to attempt an analytic solution to this problem; instead, the sample frequencies were selected by a process of trial and error. The importance of the random errors was judged from the size of the error coefficients, and that of the systematic errors from the accuracy with which the derived profile reproduced the true one. An account of the series of trials is given now .

In the search for a satisfactory set of sample frequencies, five particular cases were tried in succession. Their properties are summarized in table 1 , which gives for each case the values of the parameters $r$ and $s$, the sample frequency $f_{l}$ that corresponds to each index $l$, and the error coefficient $\rho_{l}$ of each height increment $\Delta z_{l}$ on the layer profiles. The profiles themselves were computed only for those cases where the error coefficients were reasonably small (i.e., $<10$ ), since it was found that the other cases developed severe "rounding-off errors" in the matrix multiplication (36).

\section{Cases 1 and 2}

In the first two trials a constant interval of 400 $\mathrm{kc}$ was maintained between sample frequencies. Case 1 was exactly-determined, with the number of data equal to the number of unknowns, while case 2 was overdetermined, with twice as many data as unknowns. As would be expected, the error coefficients for the overdetermined case were smaller than those for the exactly-determined case. In both cases, however, the error coefficients for the height increments in the observed frequency range were excessively large, of the order of several hundred. This fact suggests that it is not practical to space all the sample frequencies uniformly because random errors then have too severe an effect on the lower part of the deduced profile. Rather, a relatively coarse spacing must be employed in the unobserved frequency range, so that in this range the number of sample frequencies is reduced and the unknowns become very much overdetermined. 


\begin{tabular}{|c|c|c|c|c|c|c|c|c|c|c|c|c|c|c|}
\hline \multirow{3}{*}{\begin{tabular}{|c|} 
SAMPLE \\
FREQUENCY \\
$\mathrm{f} \ell(\mathrm{Mc} / \mathrm{s})$
\end{tabular}} & \multicolumn{3}{|c|}{ CASE I } & \multicolumn{3}{|c|}{ CASE 2} & \multicolumn{3}{|c|}{ CASE 3} & \multicolumn{3}{|c|}{ CASE 4} & \multicolumn{2}{|c|}{ CASE 5} \\
\hline & \multicolumn{2}{|c|}{$r=5$} & $S=5$ & \multicolumn{2}{|c|}{$r=5$} & $S=10$ & \multicolumn{2}{|c|}{$r=1$} & $S=10$ & \multicolumn{2}{|c|}{$r=3$} & $S=12$ & $r=3$ & $s=12$ \\
\hline & $\ell$ & \multicolumn{2}{|c|}{$P_{l}$} & $\ell$ & \multicolumn{2}{|c|}{$P_{l}$} & $\ell$ & \multicolumn{2}{|c|}{$P_{l}$} & $\ell$ & \multicolumn{2}{|c|}{$P_{l}$} & $\ell$ & Pl \\
\hline 0.0 & 0 & 1.9 & (2) & 0 & 1.2 & (2) & 0 & 5.1 & (0) & 0 & 6.2 & (2) & & \\
\hline 0.2 & & & & & & & & & & & & & & \\
\hline 0.4 & 1 & 4.5 & (1) & 1 & 3.7 & (2) & & & & & & & & \\
\hline 0.6 & & & & & & & & & & & & & & \\
\hline 0.8 & 2 & 1.1 & (2) & 2 & 1.9 & (2) & & & & 1 & 7.0 & (2) & 1 & $3.1 \quad(0)$ \\
\hline 1.0 & & & & & & & & & & & & & & \\
\hline 1.2 & 3 & 2.5 & (2) & 3 & 1.6 & (2) & & & & & & & & \\
\hline 1.4 & & & & & & & & & & & & & & \\
\hline 1.6 & 4 & 1.1 & (2) & 4 & 7.8 & (1) & & & & 2 & 7.2 & (1) & 2 & $4.0 \quad(0)$ \\
\hline 1.8 & & & & & & & & & & & & & & \\
\hline 2.0 & 5 & 5.3 & (0) & 5 & 3.4 & (0) & 1 & 2.7 & (0) & 3 & 2.5 & (0) & 3 & $\begin{array}{ll}1.2 & (0)\end{array}$ \\
\hline 2.2 & & & & & & & & & & 4 & 1.1 & (0) & 4 & $3.9 \quad(-1)$ \\
\hline 2.4 & 6 & 3.9 & (0) & 6 & 2.6 & (0) & 2 & 4.1 & $(-1)$ & 5 & 8.8 & $(-1)$ & 5 & $3.0 \quad(-1)$ \\
\hline 2.6 & & & & & & & & & & 6 & 7.1 & $(-1)$ & 6 & $2.4 \quad(-1)$ \\
\hline 2.8 & 7 & 2.6 & (0) & 7 & 1.7 & (0) & 3 & 3.1 & $(-1)$ & 7 & 5.8 & $(-1)$ & 7 & $2.0 \quad(-1)$ \\
\hline 3.0 & & & & & & & & & & 8 & 4.8 & $(-1)$ & 8 & $1.8 \quad(-1)$ \\
\hline 3.2 & 8 & 1.8 & (0) & 8 & 1.2 & (0) & 4 & 2.5 & $(-1)$ & 9 & 4.0 & $(-1)$ & 9 & 1.6 \\
\hline 3.4 & & & & & & & & & & 10 & 3.4 & $(-1)$ & 10 & $1.4 \quad(-1)$ \\
\hline 3.6 & 9 & 1.3 & (0) & 9 & 8.6 & $(-1)$ & 5 & 2.1 & $(-1)$ & 11 & 3.0 & $(-1)$ & 11 & $1.3 \quad(-1)$ \\
\hline 3.8 & & & & & & & & & & 12 & 2.6 & $(-1)$ & 12 & $1.2 \quad(-1)$ \\
\hline 4.0 & & & & 10 & 6.5 & $(-1)$ & 6 & 1.8 & $(-1)$ & 13 & 2.3 & $(-1)$ & 13 & 1.1 \\
\hline 4.2 & & & & & & & & & & 14 & 2.1 & $(-1)$ & 14 & $1.1 \quad(-1)$ \\
\hline 4.4 & & & & 11 & 5.1 & $(-1)$ & 7 & 1.6 & $(-1)$ & & & & & \\
\hline 4.6 & & & & & & & & & & & & & & \\
\hline 4.8 & & & & 12 & 4.1 & $(-1)$ & 8 & 1.5 & $(-1)$ & & & & & \\
\hline 5.0 & & & & & & & & & & & & & & \\
\hline 5.2 & & & & 13 & 3.4 & $(-1)$ & 9 & 1.4 & $(-1)$ & & & & & \\
\hline 5.4 & & & & & & & & & & & & & & \\
\hline 5.6 & & & & 14 & 2.9 & $(-1)$ & 10 & 1.3 & $(-1)$ & & & & & \\
\hline
\end{tabular}

TABLE 1. The error coefficients $\left(\rho_{l}\right)$ of the increments of height on the layer profile.

Each coefficient is given in the form of a number, followed in parentheses by the power of ten by which that number should be multiplied; these coefficients are dimensionless. For further explanation, see section 4 of the text.

\section{Case 3}

Accordingly, in case 3 the effect was tried of representing the entire unobserved frequency range by a single interval of sample frequency; in other words, the variation of height with plasma frequency was assumed to be linear from 0 to $2 \mathrm{Mc}$. The sample frequencies in the unobserved range, however, were the same as those in case 2 . The error coefficients now became reasonably small, and so the layer profile was computed for this case; the result is shown in figure 4. Although this profile is slightly more accurate than that of figure 3 , it still contains large systematic errors which are due to the crudity of the straight-line approximation to the layer profile in the unobserved frequency range. Evidently a representation has to be found for this part of the profile that is intermediate in complexity between those of cases 2 and 3.

\section{Case 4}

In case 4, therefore, three sample frequencies were placed in the unobserved range, as against five for case 2, and one for case 3. Also, the number of sample frequencies in the observed range was increased, and their spacing was reduced to $200 \mathrm{kc}$ in the hope that these changes would help to reduce the error coefficients. In fact, however, the error coefficients for index 0 and 1 turned out to be dis-

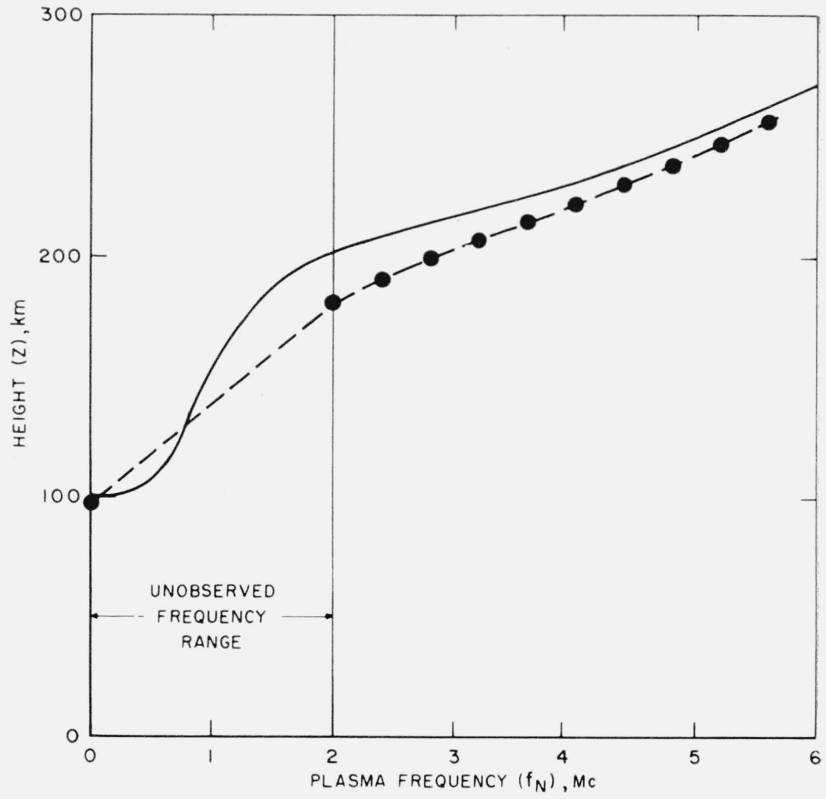

FIGURE 4. The layer profile inferred from both virtual height curves by the extended matrix method in case 3.

For further explanation, see section 4 of the text.

appointingly large. The conclusion to be drawn from this result is that no reliable detail can be obtained at the low-frequency end of the unobserved range.

\section{Case 5}

In case 5 , the final trial, the sample frequencies were the same as those in case 4 , but the ionization in the lowest interval of sample frequency was ignored: from zero frequency up to $f_{1}(0.8 \mathrm{Mc})$ the layer profile was assumed to be horizontal. This restriction was imposed on the profile by modifying the matrices $\left[M_{n m}\right]$ and $\left[\tilde{M}_{n m}\right]$. The modifications consisted of omitting the row and column with index zero, and then defining the elements of the first column to be equal to unity. These changes had the desired effect of reducing the error coefficients to acceptable values, while at the same time bringing the inferred layer profile (fig. 5) into close agreement with the true profile. The only outstanding discrepancy is at the extreme lowfrequency end of the unobserved range, where the assumption that the profile is horizontal makes it inevitable. Throughout the observed frequency range, the systematic errors of the computed points are all less than $1 \mathrm{~km}$. Hence, in this final case, the choice of sample frequencies is deemed satisfactory.

No doubt the method could be improved further by continuing this process of trial and error, but the results already achieved are felt to give a sufficient demonstration of its potentialities. 


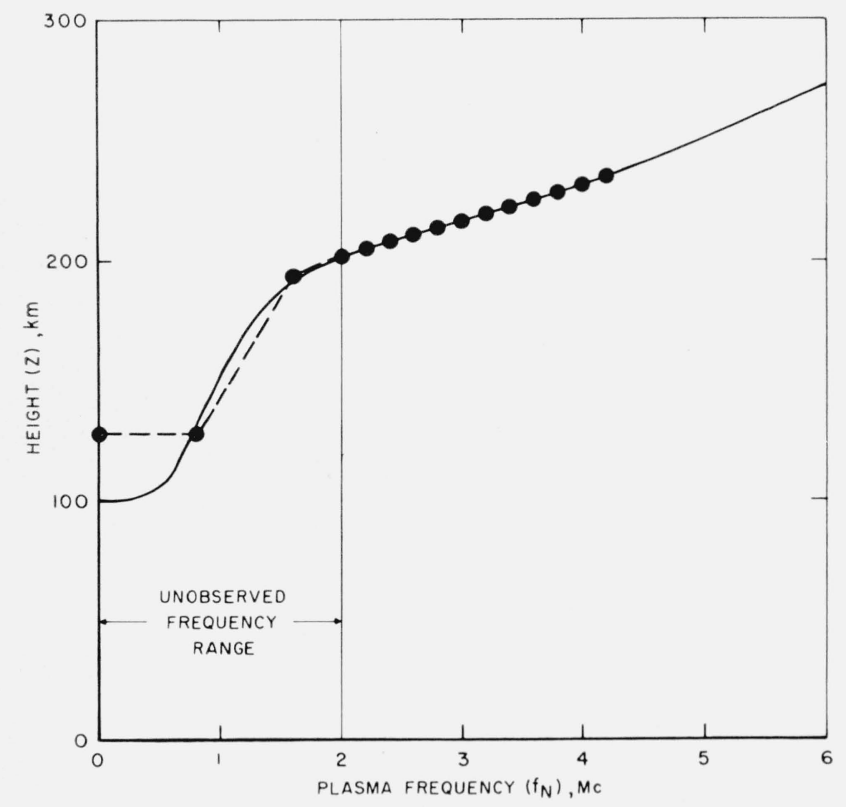

FIGURE 5. The layer profile inferred by the extended matrix method in case 5.

For further explanation, see section 4 of the text.

\section{Discussion}

This section contains a discussion of one difficulty that might be encountered in the practical use of the methods of this paper. The difficulty, noted previously in section 3.4 , is that caused by "lateral deviation." It is well known that, in vertical sounding, the paths of the ordinary and extraordinary rays within the ionosphere are deviated laterally in opposite directions [11]. So long as the ionosphere is uniform in the horizontal plane, this deviation has no effect on the virtual heights. But whenever there are horizontal gradients of electron density, then the ordinary and extraordinary rays are propagated through different distributions of ionization, and for neither mode does the virtual height curve correspond to the vertical distribution of ionization directly above the sounder. This effect has been detected already as a source of error in attempts to measure the gyrofrequency in the $F$-layer by comparison of the penetration frequencies for the two modes [11]. Presumably it also would cause errors in attempts to deduce the vertical distribution of ionization by joint use of both virtual height curves.

In this connection, it is of interest that the comparison of the penetration frequencies observed on the ionograms indicates whether or not a horizontal gradient of electron density is present. If this comparison yields the correct value for the gyrofrequency, then there can be no horizontal gradient; such an ionogram could be analyzed with confidence to obtain the vertical distribution of ionization. If, on the other hand, there is a discrepancy between the calculated and true gyrofrequencies, then the size of this discrepancy provides a measure of the gradient; perhaps this information could be used to apply some first-order correction to the virtual height curves, so as to make them more amenable to analysis.

\section{Conclusions}

In principle, given an ionogram in which the virtual heights are not recorded below some lower limiting frequency, it is possible to deduce the complete layer profile for the full range of frequency by combinining the data in the ordinary and extraordinary virtual height curves; it must be presupposed, however, that the profile is monotonic and that the data are exact.

In practice the data contain random errors of measurement, and these errors have a severe effect on the inferred profile if too much information is sought about its shape in the unobserved frequency range. To keep the effect of random errors within reasonable bounds, detail must be sacrificed in the unobserved frequency range, particularly at the lowest frequencies; this is to say that the lower part of the profile must be characterized by a few parameters only, and that these parameters must be grossly overdetermined by the data. The abandonment of this low-frequency detail involves systematic errors even when the data are exact. In fact, a balance has to be struck between random and systematic errors. It is possible to strike a balance that gives a considerable improvement over the conventional matrix method, in which the ordinary virtual-height curve alone is analyzed.

The methods need further extension to cover situations where the layer profile is multivalued.

The author is indebted to T. E. Van Zandt, E. L. Crow, S. Chapman, J. W. Wright, and J. M. Watts for discussion and advice, and to Mrs. G. A. Hessing, J. W. Finney, and R. B. Norton for assistance with the numerical computations.

\section{Appendixes}

\subsection{Appendix A: Formulas for Computing the Group Refractive Index Matrices}

The elements of the matrices $\left[M_{n m}\right]$ and $\left[\tilde{M}_{n m}\right]$ for $1 \leqq m \leqq n$, are given by the integrals in (18) and (20), respectively. These integrals cannot be evaluated analytically, in general, so they have to be evaluated numerically. As numerical integrations, however, they are difficult to perform for elements on or near the diagonal of the matrix $(m \simeq n)$, since the plasma frequency $f_{N}$ then ranges close to the critical value for reflection, where the group refractive index becomes infinite. Nonetheless, the elements themselves are all finite; and, in fact, each integral may be converted into a form that is usable right up to the diagonal by a simple change of variable [1]. 
The procedures for the ordinary and extraordinary matrices are slightly different, so they will be treated separately.

\section{a. Ordinary Matrix}

For the ordinary mode, the matrix elements are given by the integral

$$
M_{n m}=\frac{1}{\Delta f_{m}} \int_{f_{m-1}}^{f_{m}} \mu^{\prime}\left(f_{n}, f_{N}\right) d f_{N} \quad(1 \leqq m \leqq n)
$$

To obtain a form in which the integrand stays finite as $f_{N}$ approaches $f_{n}$, the variable of integration is changed to

$$
\phi=\sin ^{-1}\left(f_{N} / f_{n}\right) .
$$

Then

$$
\begin{array}{r}
M_{n m}=\frac{f_{n}}{\Delta f_{m}} \int_{\phi_{m-1}}^{\phi_{m}}\left\{\left(1-X_{n}\right)^{\frac{1}{2}} \mu^{\prime}\left(f_{n}, f_{n} \sin \phi\right)\right\} d \phi \\
\quad(1 \leqq m \leqq n),
\end{array}
$$

where $\phi_{m}, \Phi_{m-1}$ are the values of $\phi$ for which $f_{N}=f_{m}$, $f_{m-1}$, and where

$$
X_{n}=\left(f_{N} / f_{n}\right)^{2}=\sin ^{2} \phi .
$$

If the frequency interval $\Delta f_{m}$ is a constant, say $\Delta f$, so that $f_{n}=n \Delta f$, then

$$
\begin{array}{r}
M_{n m}=n \int_{\phi_{m-1}}^{\phi_{m}}\left\{\left(1-X_{n}\right)^{\frac{1}{2}} \mu^{\prime}\left(f_{n}, f_{n} \sin \phi\right)\right\} d \phi \\
(1 \leqq m \leqq n) .
\end{array}
$$

In evaluating this integral, the integrand is computed from the equations of the magneto-ionic theory, using the familiar variables

$$
\begin{gathered}
X=\left(f_{N} / f\right)^{2} \\
Y=f_{H} / f \quad Y_{L}=Y \cos \theta \quad Y_{T}=Y \sin \theta
\end{gathered}
$$

in which $\theta$ is the angle between the direction of propagation (the vertical) and the earth's magnetic field; the effect of collisions is ignored, as is usual in this type of work. From these variables, the following parameters are defined $:^{5}$

$$
\begin{aligned}
& S=\left\{Y_{T}^{4}+4 Y_{L}^{2}(1-X)^{2}\right\}^{\frac{1}{2}}, \\
& D=2(1-X)-E, \\
& E=Y_{T}^{2}+S, \\
& F=E+2 Y_{L}^{2}(1-X) .
\end{aligned}
$$

5 These parameters are similar to a set introduced by Shinn [12] who is responsible for several of the formulas quoted here. However, some of Shinn's definitions have been altered slightly.
It is convenient to compute the integrand as the product of two factors:

$$
(1-X)^{\frac{1}{2}} \mu^{\prime}=\left\{\mu \mu^{\prime}\right\}\left\{\frac{1-X}{\mu^{2}}\right\}^{\frac{1}{2}} .
$$

Here $\mu$ is the wave refractive index for the ordinary mode. Each of these factors, separately, also remains finite as $f_{N}$ approaches $f$. They are given by

$$
\mu \mu^{\prime}=\mu^{2}+\frac{X}{F^{2}}\left\{E\left(E+2 Y_{L}^{2}\right)-\frac{4 Y_{L}^{4}(1-X)\left(1-X^{2}\right)}{S}\right\}
$$

and

$$
\frac{1-X}{\mu^{2}}=1-\frac{2 Y_{L}^{2} X}{E+2 Y_{L}^{2}}
$$

while, furthermore

$$
\mu^{2}=1-\frac{E X}{F}
$$

\section{b. Extraordinary Matrix}

The integral for the elements of the extraordinary matrix is

$$
\tilde{M}_{n m}=\frac{1}{\Delta \tilde{f}_{m}} \int_{\tilde{f}_{m-1}}^{\tilde{f}_{m}} \mu_{x}^{\prime}\left(f_{n} f_{N}\right) d f_{N} \quad(1 \leqq m \leqq n)
$$

where

$$
f_{n}=\frac{1}{2} f_{H}\left[\left\{1+4\left(\tilde{f}_{n} / f_{H}\right)\right\}^{\frac{1}{2}} \pm 1\right]
$$

and the sign is to be taken positive or negative according to whether $f_{n}$ is greater or less than $f_{H}$. Equation (58) may be changed into a form suitable for numerical integration by making the substitution

$$
\tilde{\phi}=\sin ^{-1}\left(f_{N} / \tilde{f}_{n}\right) \text {. }
$$

Then

$\tilde{M}_{n m}=\frac{\tilde{f}_{n}}{\Delta \tilde{f}_{m}} \int_{\tilde{\phi}_{m-1}}^{\tilde{\phi}_{m}}\left\{\left(1-\tilde{X}_{n}\right)^{\frac{1}{2}} \mu_{x}^{\prime}\left(f_{n}, \tilde{f}_{n} \sin \tilde{\phi}\right)\right\} \tilde{d \phi}$

$$
(1 \leqq m \leqq n)
$$

where $\tilde{\phi}_{m}, \tilde{\phi}_{m-1}$ are the values of $\tilde{\phi}$ for which $f_{N}=\tilde{f}_{m}$, $\tilde{f}_{m-1}$, and where

$$
\tilde{X}_{n}=\left(f_{N} / \tilde{f}_{n}\right)^{2}=\sin ^{2} \tilde{\phi} .
$$

For the case where the frequency interval $\Delta \tilde{f}_{m}$ is a constant, say $\Delta \tilde{f}$,

$$
\tilde{M}_{n m}=n \int_{\tilde{\phi}_{m-1}}^{\tilde{\phi}_{m}}\left\{\left(1-\tilde{X}_{n}\right)^{\frac{1}{2}} \mu_{x}^{\prime}\left(f_{n}, \tilde{f}_{n} \sin \tilde{\phi}\right)\right\} d \tilde{\phi} \quad(1 \leqq m \leqq n) .
$$


Again, the integrand is computed as the product of two factors. Here the factors are, firstly,

$\mu_{x} \mu_{x}^{\prime}=\mu_{x}^{2}+\frac{2}{D}\left[1-\mu_{x}^{2}-X^{2}-\frac{\left(1-X^{2}\right)\left(1-\mu_{x}^{2}\right) Y_{L}^{2}}{S}\right]$

and secondly, the square root of

$$
\frac{1-\tilde{X}}{\mu_{x}^{2}}=\frac{D}{2(1 \mp Y)(1-X \pm Y)}\left(1+2 Y_{L}^{2} / E\right) .
$$

The wave refractive index for the extraordinary mode is given by

$$
\mu_{x}^{2}=1-\frac{2 X(1-X)}{D} .
$$

Note also that

$$
X=\tilde{X}(1 \mp Y) .
$$

Here, and in (65) above, the upper sign applies to frequencies above $f_{H}$, and the lower to frequencies below $f_{H}$.

\subsection{Appendix B: Treatment of the Overdetermined Case by the Method of Least Squares}

The equations to be solved are

$$
h_{n}^{\prime} \simeq \sum_{l=0}^{n} M_{n l} \Delta z_{l} \quad(r \leq n \leq r+s-1)
$$

for the ordinary mode, and

$$
\tilde{h}_{n} \simeq \sum_{l=0}^{n} \tilde{M}_{n l} \Delta z_{l} \quad(r \leq n \leq r+s-1)
$$

for the extraordinary. Observed values of the $h_{n}^{\prime}$ and $\tilde{h}_{n}$ are given for $r \leqq n \leqq r+s-1$, where $s \geqq r$, but these data contain random errors of measurement The aim is to find a set of $\Delta z_{l}$ which, if inserted in the equations, would yield calculated values for the $h_{n}^{\prime}$ and $\tilde{h}_{n}$ that agree with the observed values as closely as possible. The measure of disagreement is the sum of the squares of the differences between the two sets of values, and this quantity is to be minimized.

Now, if certain values are assumed for the $\Delta z_{l}$, the implied errors of measurement are

$$
E_{n}=h_{n}^{\prime}-\sum_{l=0}^{n} M_{n l} \Delta z_{l}
$$

and

$$
\tilde{E}_{n}=\tilde{h}_{n}-\sum_{l=0}^{n} \tilde{M}_{n l} \Delta z_{l} .
$$

For the ordinary mode, the sum of the squares of the implied errors is

$$
\begin{aligned}
\sum_{n=r}^{r+s-1} E_{n}^{2}=\sum_{n=r}^{r+s-1}\left(h_{n}^{\prime}\right)^{2} & -2 \sum_{n=r}^{r+s-1} \sum_{l=0}^{n} M_{n l} h_{n}^{\prime} \Delta z_{l} \\
& +\sum_{n=r}^{r+s-1} \sum_{k=0}^{n} \sum_{l=0}^{n} M_{n k} M_{n l} \Delta z_{k} \Delta_{l} .
\end{aligned}
$$

A similar expression applies to the extraordinary mode. These two expressions must be added, and the total minimized. The condition that it shall be a minimum with respect to variations of all the $\Delta z_{m}$ is that

$$
\frac{\partial}{\partial\left(\Delta z_{m}\right)} \sum_{n=r}^{r+s-1}\left\{\boldsymbol{E}_{n}^{2}+\tilde{E}_{n}^{2}\right\}=0
$$

for all $m \quad(0 \leqq m \leqq r+s-1)$. On evaluating the derivatives, this condition yields the $\Delta z_{l}$ as the solutions of (33).

\subsection{Appendix C: The Analysis Presented in Terms of the Ordinates $z_{l}$}

In the analysis described in this paper, the ordinates of the layer profile have been arrived at by two distinct steps: First, various matrix equations have been set up and solved in terms of the height increments $\Delta z_{l}$, then these increments have been summed to obtain the actual ordinates $z_{l}$. It is possible to combine these two steps by rewriting the matrix equations with the ordinates themselves as the unknowns. Consider, for instance, the eq (17) for the ordinary virtual height. It may be rearranged as follows:

$$
\begin{aligned}
h_{n}^{\prime} & \simeq \sum_{m=0}^{n} M_{n m} \Delta z_{m} \\
& \simeq M_{n 0} \Delta z_{0}+\sum_{m=1}^{n} M_{n m} \Delta z_{m} \\
& \simeq M_{n 0} z_{0}+\sum_{m=1}^{n} M_{n m}\left(z_{m}-z_{m-1}\right) \\
\simeq & M_{n 0} z_{0}+\sum_{m=1}^{n} M_{n m} z_{m}-\sum_{m=1}^{n} M_{n m} z_{m-1} \\
& \simeq \sum_{m=0}^{n} M_{n m} z_{m}-\sum_{m=0}^{n-1} M_{n, m+1} z_{m} \\
& \simeq \sum_{m=0}^{n} a_{n m} z_{m},
\end{aligned}
$$

say,

where

$$
a_{n m}= \begin{cases}M_{n m}-M_{n, m+1} & (m<n) \\ M_{n n} & (m=n) \\ 0 & (m>n) .\end{cases}
$$


This is the form in which Budden [1] uses the matrix equation; it is, of course, completely equivalent to the other.

The eq (19), for the extraordinary virtual height, may be rearranged in the same way:

$$
\tilde{h_{n}}=\sum_{m=0}^{n} \tilde{a}_{n m} z_{m}, \quad \text { say. }
$$

Since the eqs (72) and (74) are of the same form as (17) and (19), it is clear that the analysis of this paper could have been based equally well on the new equations, and developed in terms of the ordinates $z_{l}$; the arguments would proceed just as before, with the $z_{l}$ replacing the $\Delta z_{l}$, so there is no need to give the details. The choice of which of the two sets of equations to start from is largely a matter of convenience, though it may be that, in computation, one would involve smaller "rounding-off errors" than the other.

\section{References}

[1] K. G. Budden, A method for determining the variation of electron density with height $(N(z)$ curves) from curves of equivalent height against frequency $\left(\left(h^{\prime}, f\right)\right.$ curves), Rept. Cambridge Conf. Ionospheric Phys., Phys. Soc. London, p. 332 (1955).
[2] O. E. H. Rydbeck, A theoretical survey of the possibilities of determining the distribution of free electrons in the upper atmosphere, Trans. Chalmers Univ. Technol., Gothenburg, No. 3 (1942).

[3] E. R. Schmerling, The reduction of $h^{\prime}(f)$ records to electron-density-height profiles, Penn. State Univ., Ionospheric Research Sci. Rept. No. 94 (1957).

[4] J. E. Jackson, A new method of obtaining the electron density profiles from $h^{\prime}(f)$ records, J. Geophys. Research 61, 107 (1956).

[5] J. O. Thomas, The distribution of electrons in the ionosphere, Proc. I.R.E. 47, 162 (1959).

[6] J. E. Titheridge, The use of the extraordinary ray in the analysis of ionospheric records (in press).

[7] J. O. Thomas, J. Haselgrove, and A. Robbins, The electron distribution in the ionosphere over SloughI. Quiet days, J. Atmospheric and Terres. Phys. 12, 46 (1958).

[8] E. Whittaker and G. Robinson, The calculus of observations, (Blackie, London) 4th Ed., p. 209 (1944).

[9] F. B. Hildebrand, Introduction to numerical analysis, p. 323 (McGraw-Hill Book Co., Inc., New York, N.Y., 1956).

[10] J. D. Chappell, Double precision matrix inversion, IBM 650 Program Library, File No. 5.2.009.

[11] J. C. W. Scott, Longitudinal and transverse propagation in Canada, J. Geophys. Research 55, 65 (1950).

[12] D. H. Shinn, Tables of group refractive index for the ordinary ray in the ionosphere, Rept. Cambridge Conf : Ionospheric Phys., Phys. Soc. London, p. 402 (1955).

Boulder, Colo.

(Paper 64D2-45) 